\title{
Perioperative hypothermia during surgery: is warming humidification a complete solution?
}

\author{
Antonio M. Esquinas \\ Intensive Care Unit, Hospital Morales Meseguer, Murcia, Spain
}

Hypothermia is a common complication in postoperative patients with well-known effects on hospital outcome [1]. Patients undergoing shoulder arthroscopy surgery in some cases develop different grades of perioperative hypothermia as a secondary effect of cold shoulder irrigation. Additionally, sedation and invasive mechanical ventilation aggravate this state. However, published studies regarding rational pathways to hypothermia during arthroscopic surgery suggest that other mechanisms could be influencing the heat loss, such as dry gases. Commonly, humidification devices, referred to as "heat and moisture exchangers" or "humidified and electrically heated circuits" (HHC), are used to - indirectly - control core body temperature (CBT), but this issue remains controversial when operating under invasive mechanical ventilation, and the efficacy remains unclear [1].

Jo et al. [2] described the effects of two humidification devices during arthroscopic shoulder surgery; the working hypothesis was that active warming and humidification of inspired gases may influence the decrease in CBT in arthroscopic shoulder surgery. The authors analyzed two devices, a conventional respiratory circuit (control group) and heating using a HHC (heated group). The major findings were significant temperature differences in CBT during two periods, immediately after anesthesia induction (30 min), and then in the HHC group, CBT was maintained better from 75 to 120 min after anesthesia induction. This original study makes a considerable contribution and has practical, clinical implications. However, other pathways may also explain the results and should be taken into account by the authors.

First, a key aspect to consider is that the hypothermia re- ported could depend, in part, on lung function and mechanical ventilation interactions during arthroscopic surgery. Although the authors enrolled patients without previous lung disease, they did not provide complete information regarding the mechanical ventilation parameters. Briefly, these interactions depend mainly on the total flow rate during general anesthesia. Some studies reported that with a total flow rate of $2 \mathrm{~L} / \mathrm{min}$ for $2 \mathrm{~h}$, a humidifier might not be necessary, while with a total flow of $4 \mathrm{~L} / \mathrm{min}$, it could be useful to maintain inspired gas humidity [3]. A secondary contributing factor is minute volume (MV) during surgery, especially if we consider the MV upper limit to be $7 \mathrm{~L} / \mathrm{min}$ [4]. These ventilator parameters are unknown and are critical issues.

Second, the authors use a "critical time" (after $75 \mathrm{~min}$ ) to compare differences between the groups. However, this period was not fully justified by the authors and others have used a greater critical time period, of 14-h duration, to observe differences in humidification and BCT [5]. It is important to know whether the parameters of the mechanical ventilation and surgical procedure were stable during this period to exclude these factors. In this case, it seems more reasonable that differences between the two groups were associated with modifications in lung humidification function and reduced water loss from the airways because of the HHC. These aspects are essential for a proper analysis.

Further large prospective clinical trials are needed to determine the interactions of humidification devices during arthroscopic surgery to avoid a hypothermic state, focusing on high-risk surgical patients and patient-mechanical ventilator interactions.

Corresponding author: Antonio M. Esquinas, M.D., Ph.D., Intensive Care Unit, Hospital Morales Meseguer, Avenida Marques de los Velez, s/n, Murcia, 3008, Spain. Tel: 34-609321966, Fax: 34-968232484, E-mail: antmesquinas@gmail.com

(c) This is an open-access article distributed under the terms of the Creative Commons Attribution Non-Commercial License (http:// creativecommons.org/licenses/by-nc/3.0/), which permits unrestricted non-commercial use, distribution, and reproduction in any medium, provided the original work is properly cited. 


\section{References}

1. Leslie K, Sessler DI. Perioperative hypothermia in the high-risk surgical patient. Best Pract Res Clin Anaesthesiol 2003; $17: 485-98$.

2. Jo YY, Kim HS, Chang YJ, Yun SY, Kwak HJ. The effect of warmed inspired gases on body temperature during arthroscopic shoulder surgery under general anesthesia. Korean J Anesthesiol 2013; 65: 14-8.

3. Yamashita K, Yokoyama T, Abe H, Nishiyama T, Manabe M. Efficacy of a heat and moisture exchanger in inhalation anesthesia at two different flow rates. J Anesth 2007; 21: 55-8.

4. Rathgeber J, Weyland W, Bettka T, Züchner K, Kettler D. Is reduction of intraoperative heat loss and management of hypothermic patients with anesthetic gas climate control advisable? Heat and humidity exchangers vs. active humidifiers in a functional lung model. Anaesthesist 1996; 45: 807-13.

5. Goldberg ME, Epstein R, Rosenblum F, Larijani GE, Marr A, Lessin J, et al. Do heated humidifiers and heat and moisture exchangers prevent temperature drop during lower abdominal surgery? J Clin Anesth 1992; 4: 16-20. 